\title{
Main technical features and performance evaluation of a miniaturized test system for star trackers
}

V. Nardino, M. Burresi, M. Cecchi, T. Cecchi, F. Corti, et al.

V. Nardino, M. Burresi, M. Cecchi, T. Cecchi, F. Corti, E. Franci, G. Guidotti, D. Guzzi, C. Lastri, L. Palombi, L. Salvadori, C. Spagnesi, Valentina Raimondi, "Main technical features and performance evaluation of a miniaturized test system for star trackers," Proc. SPIE 11852, International Conference on Space Optics - ICSO 2020, 1185260 (11 June 2021); doi: $10.1117 / 12.2600309$

SPIE Event: International Conference on Space Optics - ICSO 2021, 2021, Online Only 


\section{International Conference on Space Optics-ICSO 2020}

Virtual Conference

30 March-2 April 2021

Edited by Bruno Cugny, Zoran Sodnik, and Nikos Karafolas
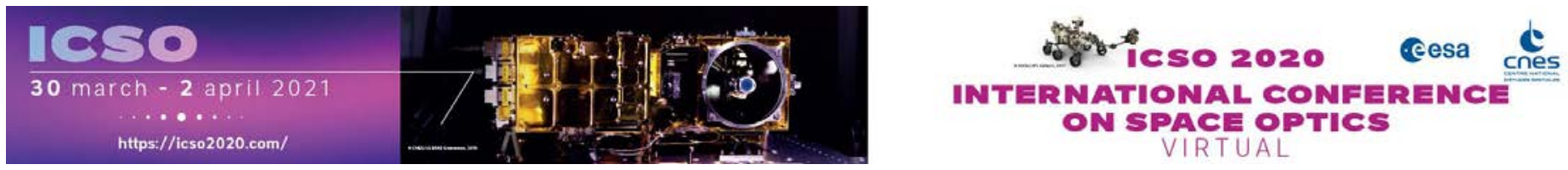

\section{Main technical features and performance evaluation of a miniaturized test system for star trackers}

\section{Cesa isoporecestings denes}




\title{
Main technical features and performance evaluation of a miniaturized test system for star trackers
}

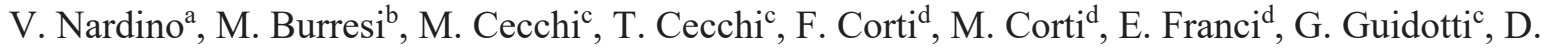 \\ Guzzi $^{\mathrm{a}}$, C. Lastri ${ }^{\mathrm{a}}$, L. Palombi ${ }^{\mathrm{a}}$, L. Salvadori ${ }^{\mathrm{d}}$, C. Spagnesi ${ }^{\mathrm{b}}$, V. Raimondi*a \\ a"Nello Carrara" Institute of Applied Physics (IFAC) - National Research Council (CNR), Via \\ Madonna del Piano, 10 - 50019 Sesto Fiorentino (Firenze, Italy); ${ }^{b}$ Gestione Silo S.r.l., Via Di

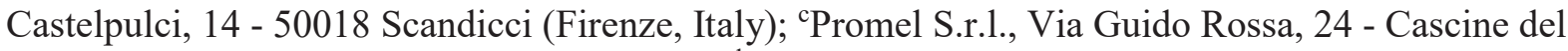 \\ Riccio - 50023 (Impruneta, Firenze, Italy); ${ }^{\mathrm{d}}$ Saitec S.r.1. - Via Fiorentina 17/D - 50063 Figline \\ Valdarno (Firenze, Italy)
}

\begin{abstract}
The validation of the correct functioning of star trackers is a crucial step for the success of any space mission. In recent years, there has been an increasing interest for the development of miniaturized, light-weighted test systems to be installed directly on the star tracker, opening the way to the test of completely assembled, on-board attitude sensors in pre-launch environment.

Here we present the main technical characteristics of a new prototype, the MINISTAR, recently developed by a consortium of Italian enterprises and the Applied Physics Institute of the National Research Council. Besides its main technical features, here we present the results of a set of tests for its performance evaluation, with particular reference to its radiometric and geometric calibration in the laboratory. The MINISTAR can generate synthetic images of dynamic star fields for the simultaneous test of multiple star trackers (up to three head devices). The generation of dynamic star fields in a realistic scenario also includes large objects, such as the Sun or the Moon, and disturbances (e.g. cosmic rays and stray light effects).

Thanks to its reduced dimensions and weight, the MINISTAR is suitable for the installation on the star tracker's baffle. Special attention has also been paid to the use of materials and technologies that could be compliant for vacuum operation in the future. Thanks to the design and construction of an interchangeable mechanical interface, the MINISTAR is compatible with the majority of star trackers available on the market.
\end{abstract}

Keywords: Star Tracker, OGSE, Miniaturized device, Test system, Star fields, Attitude control, Open loop, Closed loop.

\section{INTRODUCTION}

Star trackers are electro-optical devices that are fundamental for determining the attitude of satellite platforms in the frame reference. Satellite orientation with respect to the frame reference of fixed star is obtained from the image of the observed star field. Being a critical element for every satellite mission, usually a redundant number of star trackers (typically 3 ) is mounted onboard of a platform, in order to guarantee a continuous availability of satellite attitude data, both in case of failure and partial/total field of view occupation by large objects, such as the Earth or the Moon. The validation of the star trackers operation is a fundamental step for any space mission.

The test of star trackers is typically carried out by means of Optical Ground Support Equipment (OGSE) that are used to test both the optical characteristics of the star trackers and their internal algorithm for star recognition, and thus to verify the performance of the system as a whole. Most OGSE are designed for testing star trackers performance in laboratory environment. Instead, their validation after onboard installation requires both the simulation of dynamic star fields and device miniaturization, in order to install it directly on the star tracker. The availability of such devices on the market is still very limited.

*v.raimondi@ifac.cnr.it; phone +39 055522 6379; www.ifac.cnr.it/corsari/meteors 
Most OGSE tests star trackers performance in the laboratory, before their installation on the platform. These devices present a static or a dynamic simulation of the scene observed by the star tracker so that the output of the star tracker can be compared with the input data provided by the OGSE [1,2]. Some laboratory facilities rely on the use of laser or light emitting diodes (LEDs) to mimic a static scene that portraits the brightest stars of the night sky [3]. Another approach is the one that relies on the use of a large LCD screen showing the simulated star-field viewed by the star sensor. In this case, the laboratory facility can perform either static or dynamic simulations. All these methods are based on some simplifications that can limit the accuracy of the test. Efforts have been made to overcome some of these limitations, such as the radiometric modulation of the source used in the simulation [4].

The validation of star trackers after their assembly on the platform is always challenging. It requires the implementation of test systems able to simulate dynamic star fields as those observed during the on-board operational activity and the development of miniaturized test systems to be installed directly on the star tracker. The availability on the market of such miniaturized device is still very limited. Presently, only few miniaturized devices are available, e.g. the STOS (Star Tracker Optical Stimulation for Sensors) manufactured by Airbus Space Equipment [5] and the Optical Sky Stimulator (OSI) for ASTRO APS star sensors by Jena-Optronik [6]. Both systems simulate a dynamic scene of a star field. These devices use a miniaturized LCD monitor showing the simulated image in apparent motion to the star tracker and an optical system projecting the simulated image in the star tracker optics. These systems can simulate large non-star objects and different disturbances (like stray light and the effects due to charged particles). They can be used either in open-loop configuration for sensor test or in close-loop configuration to test the behavior of the star tracker operating in a realistic scenario.

In this paper, we present a new OGSE prototype, the MINISTAR, recently developed by a consortium of Italian enterprises and the Applied Physics Institute of the National Research Council. The MINISTAR is a miniaturized electro-optical device able to generate synthetic images of dynamic star fields. Its design permits the simultaneous test of multiple star trackers, both for optics, electronics and on-board attitude software since it performs a dynamic simulation of the apparent motion of the observed scene. It can simulate the presence of large objects, custom objects and disturbances such as cosmic rays and stray light effects. MINISTAR can be placed directly on the star tracker under test. Besides its main technical features, here we present the results of a set of tests for its performance evaluation, with particular reference to its radiometric and geometric calibration in the laboratory.

\section{THE MINISTAR PROTOTYPE}

\subsection{Prototype's description}

Ministar system architecture, depicted in Figure1, is made up of the following sub-systems:

- Star field simulation software. The software receives, as input, the attitude of the satellite platform and, for each star tracker head under test, the field of view and the view direction. It produces as output the dynamic scene of the simulated star field for each star tracker, using the magnitude and positions of the stars of the HIPPARCOS [7] star catalogue.

- Opto-mechanical system. The simulated star field is projected in the star tracker's field of view by means of a collimating optical system. The opto-mechanical assembly permits an accurate coupling between the MINISTAR device and the star tracker.

- $\quad$ Processing unit and electronics. This module performs the test procedure allowing the control of the simulation and the rendering on the MINISTAR display of the synthetic star field scene. The correct operation of the MINISTAR is verified by the processing unit.

The comparison of the simulated data by MINISTAR and the star tracker output allow the test and the validation of the star tracker.

Figure 2 shows the assembled MINISTAR prototype (Figure 2A) and the detail of monitor with the rendered star field (Figure 2B). 


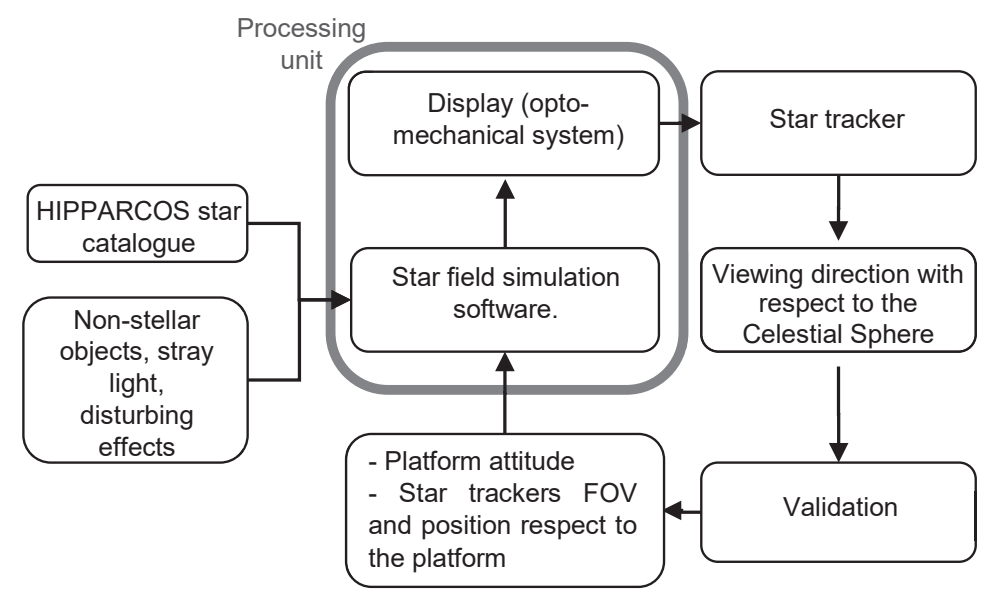

Figure 1. Block diagram of the MINISTAR system showing the main elements of a simulation in closed-loop configuration for validation of the star tracker under test.

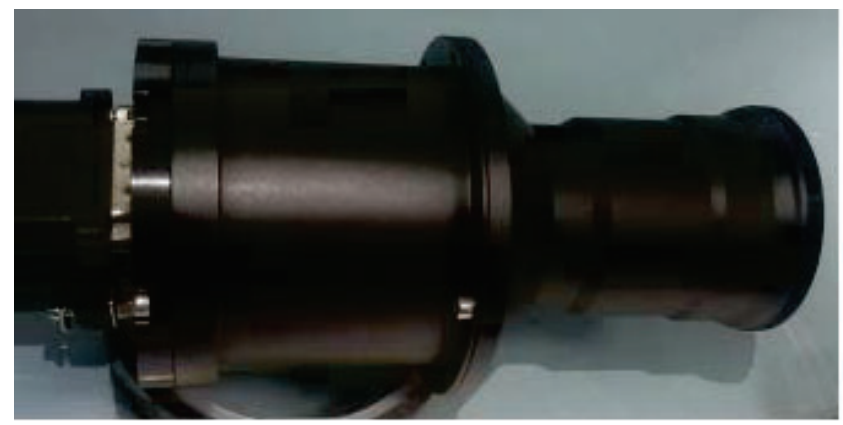

A

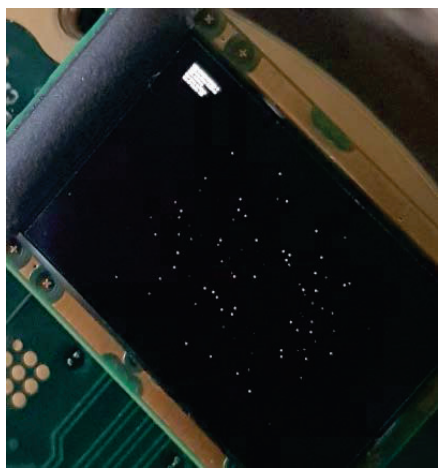

B

Figure 2. The MINISTAR: (A) the assembled prototype, and (B) a detail of the monitor with the rendered star field.

\subsection{Main technical features of the prototype}

The MINISTAR design requires a fast computation, system miniaturization, and the capability of testing different types of star trackers commercially available.

The request for a computationally fast algorithm lead to the choice of a modular structure for the design of the simulation model and control interface. The MINISTAR simulation model provides a real time computation of the dynamic synthetic scenes and also permits the simulation of non-stellar objects and other disturbance effects such as stray light or SEU (Single Event Upset). Such model also manages the rendering of the selected scenario on the display for custom Field of View (FOV) and radiometric dynamics.

MINISTAR is controlled through a graphic user interface that permits the selection, for the simulation, of the desired star catalogue (either HIPPARCOS [7] or different custom star catalogues) and user-selected non-star objects. The software was also designed for the simultaneous test of multiple-head star tracker. The following figure shows the operational scheme of the MINISTAR software model for star field rendering. 


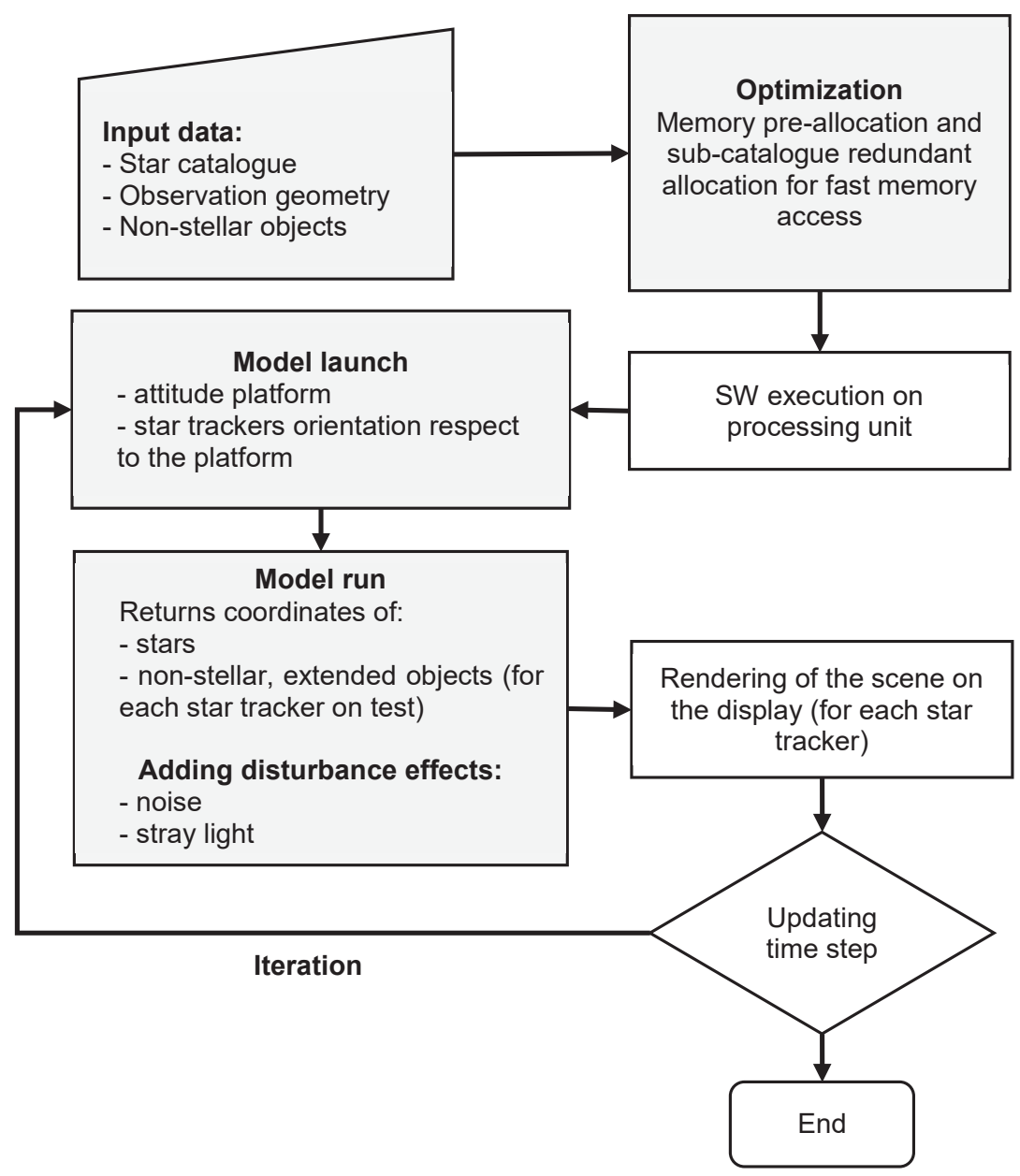

Figure 3. MINISTAR software scheme showing the optimization phase of the mathematical model (at allocation time) followed by the model launch. This structure increases computational speed.

Miniaturization of the device was another key for the mechanical design of MINISTAR. Its construction was carried out using innovative materials to obtain a light-weight, miniaturized opto-mechanical assembly characterized by a weight less of $1 \mathrm{~kg}$ and dimensions of $175 \mathrm{~mm}$ ( ) x $150 \mathrm{~mm}$. These characteristics makes it possible the mounting directly on the star tracker's baffle.

MINISTAR was designed to be compliant with several star tracker models available on the market, for this reason it has a large pupil and FOV in order to be compatible with most of the star trackers.

Table 1 shows the main technical data of the MINISTAR prototype. 
Table 1. MINISTAR technical features

\begin{tabular}{|ll|}
\hline Stimulation & Functional testing of Star Tracker \\
\hline Display dimensions & $1920 \times 1200$ pixels \\
\hline FOV & $20^{\circ}\left( \pm 10^{\circ}\right)$ \\
\hline Frame rate for dynamic scene & $85 \mathrm{~Hz}$ \\
\hline Pupil diameter & $35 \mathrm{~mm}$ \\
\hline Device dimensions & $86 \mathrm{~mm}(\varnothing) \times 150 \mathrm{~mm}(175 \mathrm{~mm} \varnothing \times 150 \mathrm{~mm}$ with baffle flange $)$ \\
\hline Weight & $<1 \mathrm{Kg}$ \\
\hline Alignment error & $0.001^{\circ}$ \\
\hline Single Star Accuracy & $0.005^{\circ}$ \\
\hline Star Catalogue & $\mathrm{HIPPARCOS}$ \\
\hline Star magnitude range & $4.5 \pm 0.2 \mathrm{mag}$ \\
\hline
\end{tabular}

\section{PERFORMANCE EVALUATION}

MINISTAR was tested in laboratory for characterizing radiometric and geometric performance. The experimental tests used two cameras: DALSA 1M60 camera with 45-mm NIKON objective panchromatic camera for performing radiometric tests, Canon SX60 HS set at 85-mm equivalent focal length commercial camera for MINISTAR distortion characterization. Figure 4 shows the setup used for MINISTAR laboratory tests.

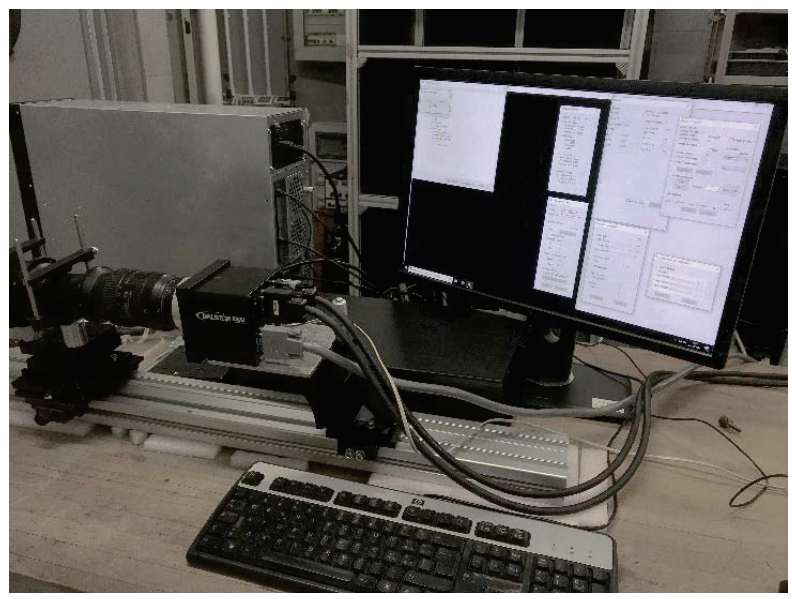

Figure 4. MINISTAR prototype during laboratory tests. On the left, the prototype faced to the objective and the camera used for the test (DALSA, 1M60 model).

DALSA 1M60 with its objective was radiometrically characterized and calibrated using the calibrated Lambertian source HL-3P-INT-CAL with double diffuser, while Canon SX60 HS camera was geometrical characterized using geometric patterns at fixed distance as truth. After their characterization and calibration, both cameras were used to characterize the MINISTAR response. 


\subsection{MINISTAR Radiometric calibration}

For the generic pixel of the image obtained by a camera observing a generic radiance source $L(\lambda)$ we expect the value (expressed in digital numbers $D N$ ):

$$
D N=k \tau\langle L\rangle_{\Delta \lambda}
$$

where $\langle L\rangle_{\Delta \lambda}$ is the radiance (Lambertian) expressed in $\mathrm{Wm}^{-2} \mathrm{sr}^{-1} \mathrm{~nm}^{-1}$ averaged in the spectral channel $\Delta \lambda$ between $\lambda_{0}$ and $\lambda_{1}, \tau$ is the integration time, $k$ is a constant depending on the camera entrance pupil area, focal length, pixel dimensions and optoelectronics parameters. The spectral average $\langle L\rangle_{\Delta \lambda}$ on a particular spectral channel is expressed in $\mathrm{Wm}^{-2} \mathrm{sr}^{-1} \mathrm{~nm}^{-1}$ and is given by the operator of integral average:

$$
\langle L\rangle_{\Delta \lambda}=\int_{\lambda_{0}}^{\lambda_{1}} S(\lambda) L(\lambda) d \lambda
$$

With $S(\lambda)$ being the spectral response of the sensor, integrally normalized to unit in the spectral interval $\Delta \lambda=\lambda_{1}-\lambda_{0}$ and null elsewhere.

The radiometric calibration of the MINISTAR consists in determining the constant $k$ for the calibration camera and radiometrically characterizing the spectrum of the generic pixel of MINISTAR display.

For determining the value of $k$, the image of a VIS-NIR radiometrically calibrated Lambertian source HL-3P-INT-CAL with double diffuser is acquired with a DALSA 1M60 camera equipped with a NIKON 45 mm objective set to infinity. In the hypothesis of Lambertian emission, in the pixels of the source image, we have a calibrated (truth) average value $D N_{\text {truth }}$ given by:

$$
D N_{\text {truth }}=k \tau\left\langle L_{\text {truth }}\right\rangle_{\Delta \lambda}+D N_{\text {tark }}
$$

with $D N_{\text {dark }}$ being the dark average value, read with $L_{\text {truth }}(\lambda)=0$. truth $\left\langle L_{\text {truth }}\right\rangle_{\Delta \lambda}$ is calculated for the DALSA using its detector spectral response $S_{D}(\lambda)$ (from the datasheet) convolved with the spectrum $L_{\text {truth }}(\lambda)$ of the calibrated source. Such convolution can be numerically determined and, by the knowledge of $\left\langle L_{\text {truth }}\right\rangle_{\Delta \lambda}, D N_{\text {truth }}$ and $D N_{\text {dark }}$, the calibration constant $k$ (expressed in $\frac{D N}{J m^{-2} s r^{-1} \mathrm{~nm}^{-1}}$ ) can be determined and used to characterize the spectrum of the MINISTAR display.

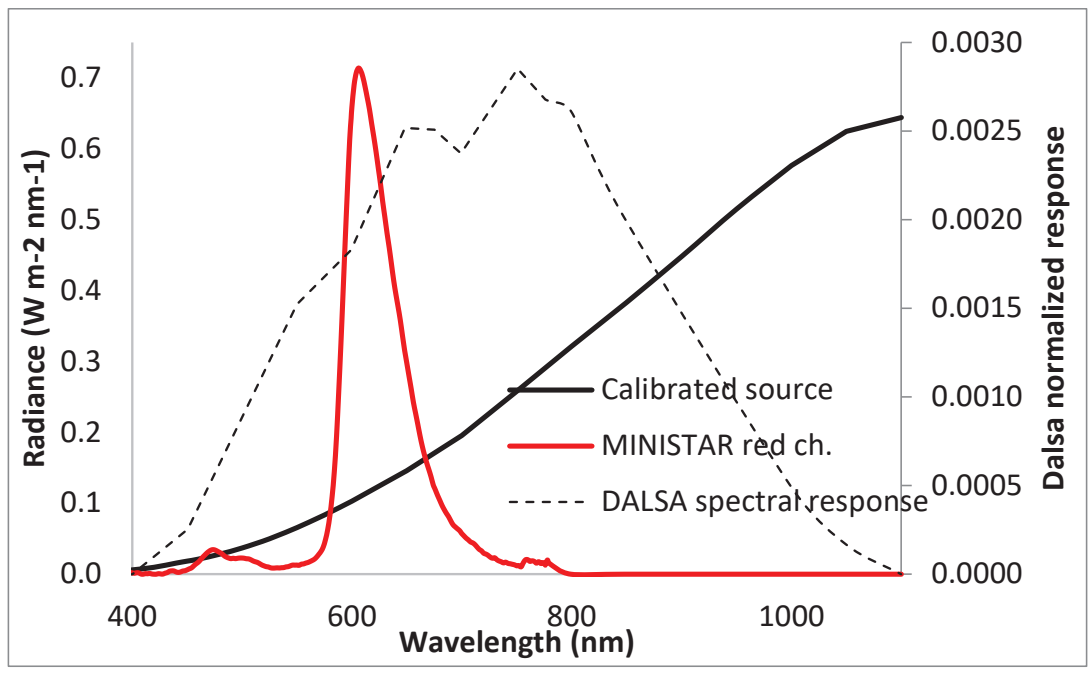

Figure 4. Spectral radiance of the MINISTAR channel used for the simulation (in our case the R channel) $\left\langle R r_{M S}\right\rangle_{\Delta \lambda}$ (in red) and of the calibrated source HL-3P-INT-CAL equipped with Lambertian diffuser (in black), together with the DALSA 1M60 integrally normalized spectral response (dashed).

Each MINISTAR pixel emits the light spectrum of its RGB OLED display in the colour channel selected for the simulation (in our case the red channel R). Even if the spectral shape of the OLED display is known from its datasheet, it has to be radiometrically characterized. We can consider the OLED red channel spectrum as the product of a normalized 
adimensional spectral response $r_{M S}(\lambda)$ (from the datasheet) and a factor $R$ with dimensions of $W^{-2} s r^{-1} \mathrm{~nm}^{-1}$. For radiometrically characterizing the OLED spectrum, $R$ has to be determined.

The acquisition performed using the MINISTAR screen as extended, constant light source (with screen brightness set to a user-defined fixed value) brings to an average signal $D N_{M S}$ for the generic pixel given by:

$$
D N_{M S}=k \tau\left\langle R r_{M S}\right\rangle_{\Delta \lambda}+\underset{M S}{D N_{d a r k}}
$$

with $D N_{\text {dark }}$ being the dark value, read with empty (black) display.

As in the case of the calibrated source, the spectrally averaged radiance measured by the DALSA detector is:

$$
\left\langle R r_{M S}\right\rangle_{\Delta \lambda}=\frac{D N_{M S}-D N_{\text {dark }}}{k \tau}=R \int_{\Delta \lambda} S_{D}(\lambda) r_{M S}(\lambda) d \lambda
$$

By calculating the value of $\int_{\Delta \lambda} S_{D}(\lambda) r_{M S}(\lambda) d \lambda$ and measuring the difference $D N_{M S}-D N_{d a r k}$, the value of the constant $R$ can be determined as:

$$
R=\frac{D N_{M S}-D N_{\text {dark }}}{k \tau \int_{\Delta \lambda} S_{D}(\lambda) r_{M S}(\lambda) d \lambda}
$$

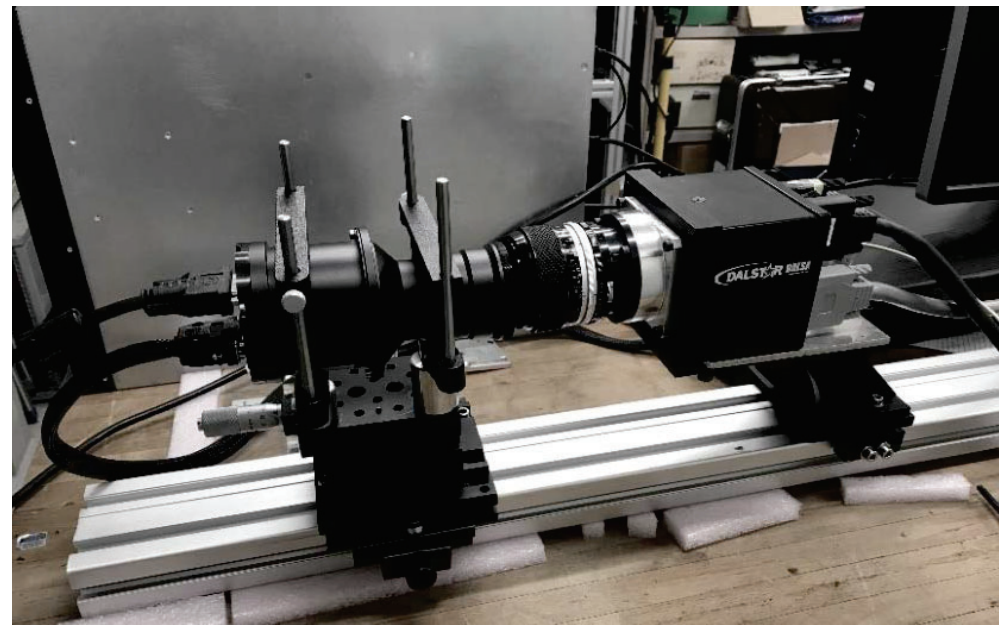

Figure 5. Setup for radiometric calibration: MINISTAR is faced to the NIKON objective coupled to the DALSA camera.

In Figure 5 the spectral radiance of the MINISTAR channel used for the simulation (in our case the R channel) $\left\langle R r_{M S}\right\rangle_{\Delta \lambda}$ the calibrated source HL-3P-INT-CAL equipped with Lambertian diffuser, together with the DALSA 1 M60 integrally normalized spectral response are reported.

A first laboratory test was the verification of MINISTAR radiometric response linearity. Figure 6 shows the laboratory set up for radiometric tests.

In Figure 7 the acquired digital number versus the display relative luminosity is reported.

A second test was the verification of MINISTAR ability to reproduce the exact magnitude of the stars. Figure 8 shows the comparison of the star magnitude in Cassiopeia constellation simulated by MINISTAR compared with the visual magnitude of the same stars listed in HIPPARCOS catalogue. The agreement is very satisfactory. 


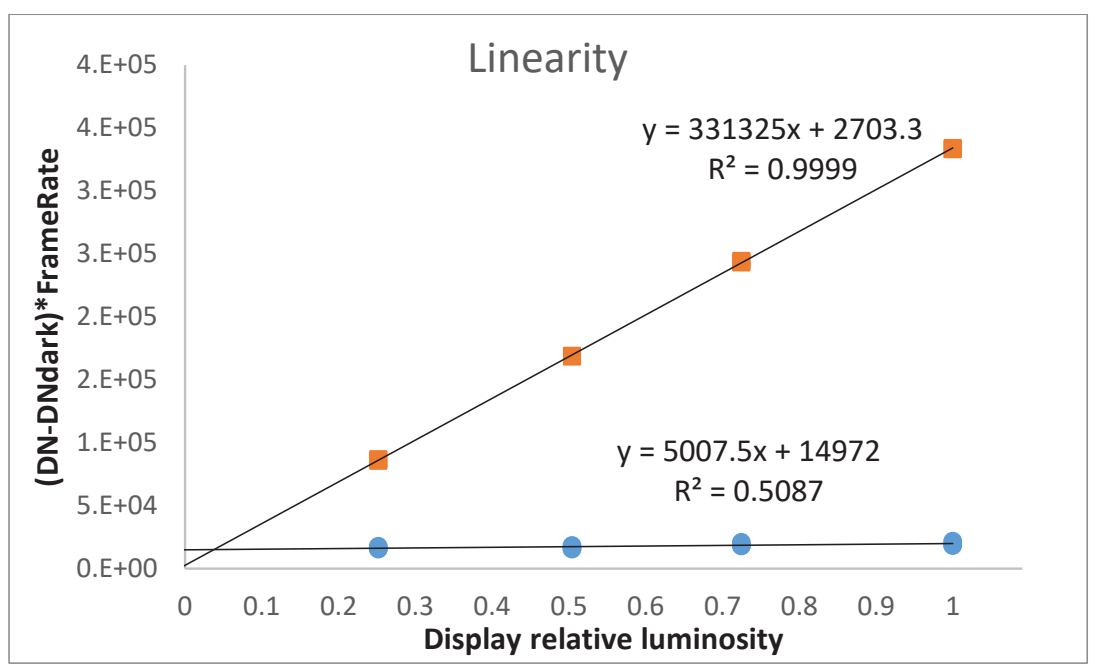

Figure 6. Verification of linearity in radiometric response in DN of the MINISTAR display as a function of its relative luminosity.

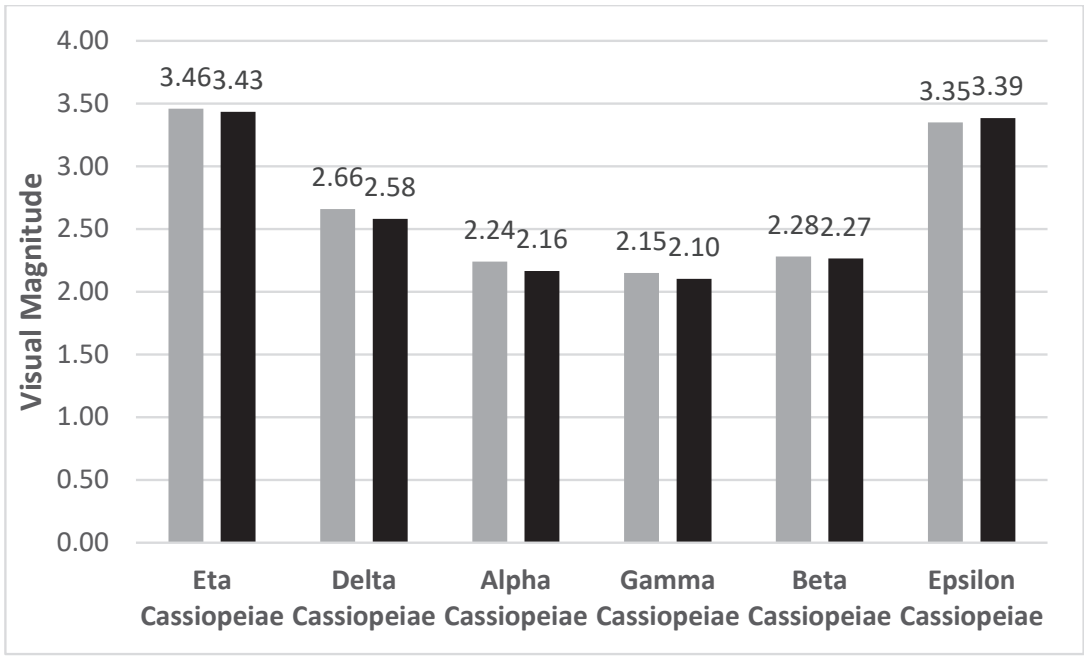

Figure 7. Ministar-simulated (gray) vs HIPPARCOS (black) star visual magnitude comparison.

\subsection{MINISTAR Geometric calibration}

Geometric calibration involved the use of a high resolution camera. The following figure shows the laboratory setup for geometrical tests.

For geometric tests the following two-step procedure was adopted:

1) verification that the camera Canon SX60 HS set at $85 \mathrm{~mm}$ equivalent focal length used for measurement can be considered distortion-free (i.e. the errors on angles have to be less than MINISTAR angular uncertainty);

2) distortion-free camera is used to measure the (distorted) position of a grid of regularly distributed points (truth grid) on the MINISTAR field of view. 
The first step was performed by acquiring the image of a regularly aligned pattern acquired at resolution of $4608 \times 3456$ pixels. From geometric considerations on the dimension of the test pattern and its distance, the angular span of a pixel can be determined. In particular, we made sure that the maximum deviation (in pixels) with respect of a horizontal and vertical grid was less than MINISTAR uncertainty on star angular position ( 0.005 degrees), so that the camera image can be used for measuring the MINISTAR angular distortion map.

MINISTAR geometric characterization was performed displaying a chessboard on MINISTAR display (acquired with corrected camera) and verifying the distortion in the acquired image.

The inversion of the relation above allows the software correction of MINISTAR optical system deformation. Inverted formula is obtained by fitting two analogous polynomial surfaces $x\left(x_{M}, y_{M}\right)$ and $y\left(x_{M}, y_{M}\right)$ on the distorted coordinates. The obtained formula returns the coordinates $x, y$ to be set on the display corresponding to a pixel at coordinates $x_{M}, y_{M}$ on the MINISTAR exit pupil. The RMS error between the fitted formula and the distortion induced on the coordinates by the MINISTAR optics is 0.51 pixels.

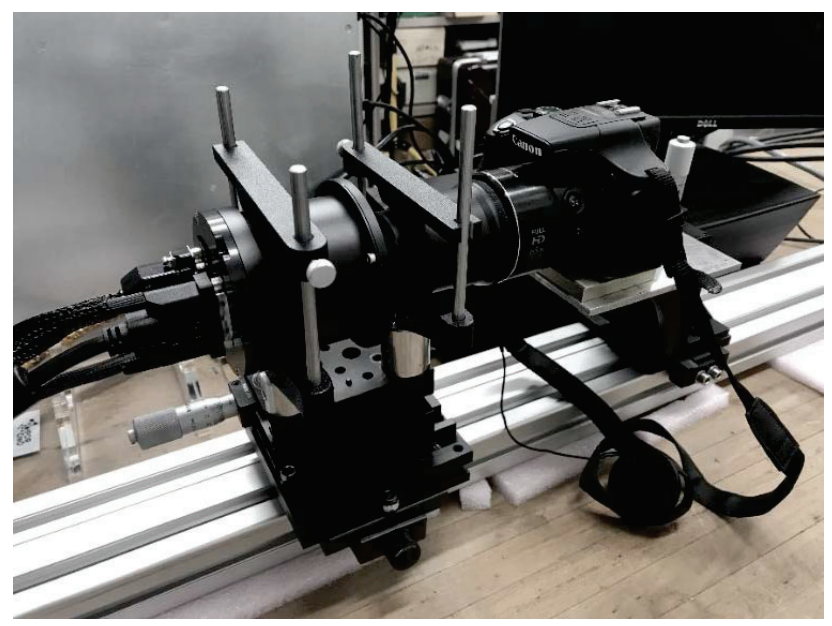

Figure 8. Laboratory setup for geometric calibration: MINISTAR (left) is faced to Canon SX60 HS camera.

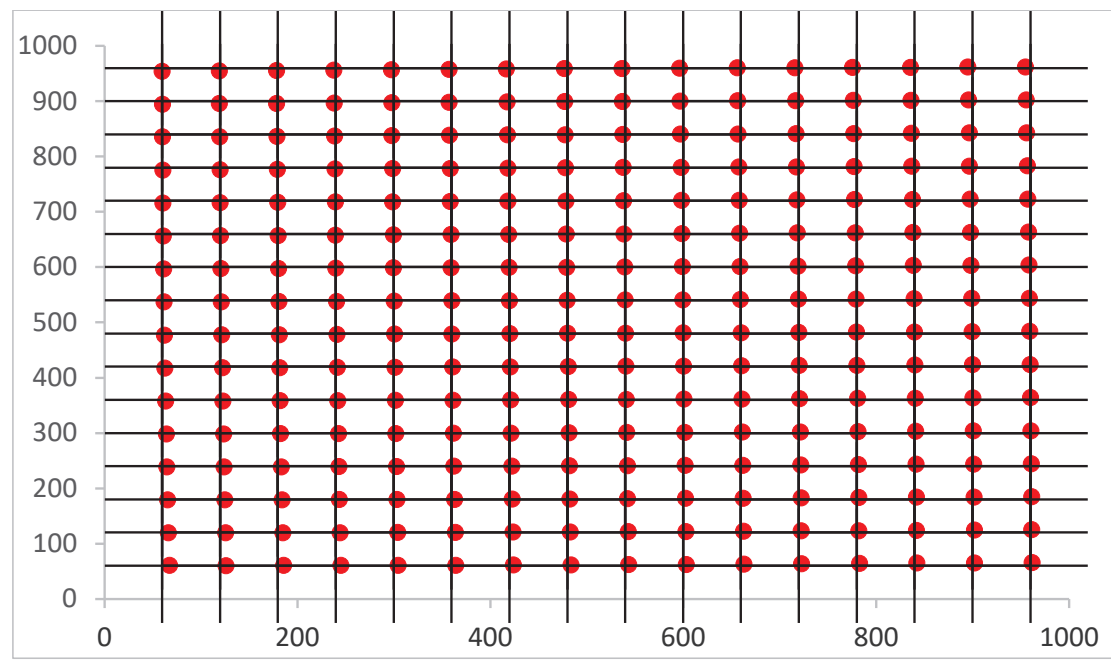

Figure 9. Coordinates of MINISTAR distorted pixel (red) compared to the ideal grid (in black) having the nodes in the ideal pixel center. 


\section{CONCLUSIONS}

We presented a novel, miniaturized test system - the MINISTAR - for star trackers. MINISTAR offers the possibility to test simultaneously the performance of the optical, electronic and onboard attitude software of the star tracker. In addition, its small weight and dimensions allows the installation directly on the star tracker under test. MINISTAR can test multihead systems (up to three head devices) by generating dynamic star fields in a realistic scenario that includes large objects like the Sun or the Moon, custom objects and disturbances. The performance tests made in the laboratory showed that MINISTAR is able to reproduce the magnitude of the stars and that the images shown on MINISTAR display are not affected by strong distortions.

\section{REFERENCES}

[1] Rufino G., Accardo D., Grassi M., Fasano G., Renga A., and Tancredi U., "Real-Time Hardware-in-the-Loop Tests of Star Tracker Algorithms,” International Journal of Aerospace Engineering, 505720 (2013).

[2] Yang J., Liang B., Zhang T., Song J., Song L., "Laboratory Test System Design for Star Sensor”, Journal of Computers 7(4), $1056-1063$ (2012).

[3] Boone B. G., Bruzzi J. R., Dellinger W. F., Kluga B. E., and Strobehn K. M., "Optical Simulator and Testbed for Spacecraft Star Tracker Development”, Optical Modeling and Performance Predictions II, 586711, (2005).

[4] Dzamba T. and Enright J., "Ground Testing Strategies for Verifying the Slew Rate Tolerance of Star Trackers", Sensors, 14, 3939-3964 (2014).

[5] https://airbus604.rssing.com/chan-59901147/all_p1.html (last accessed February 16, 2021).

[6] Samaan M.A., Stees S. R., and Theil S., "Star Tracker Real-Time Hardware in the Loop Testing Using Optical Star Simulator," Spaceflight Mechanics, 140 (2011).

[7] Perryman M. A. C., Lindegren L., Kovalevsky J., Hoeg E., Bastian U., Bernacca P. L., Crézé M., Donati F., Grenon M., Grewing M., van Leeuwen F., van der Marel H., Mignard F., Murray C.A., Le Poole R. S., Schrijver H., Turon C., Arenou F., Froeschlé M., Petersen C. S., "The HIPPARCOS Catalogue, Astronomy and Astrophysics, Vol. 323, p.L49-L52 (1997). 